\title{
Flexible Textile Direct-Current Generator Based on the Tribovoltaic Effect at Dynamic Metal- Semiconducting Polymer Interfaces
}

Jia Meng, ${ }^{a, b}$ Zi Hao Guo, , ${ }^{b, c}$ Chongxiang Pan, ${ }^{a, b}$ Luyao Wang, ${ }^{a, b}$ Caiyun Chang, ${ }^{a, b}$ Longwei Li, ${ }^{b, c}$ Xiong Pu ${ }^{a, b, c, *}$ and Zhong Lin Wang ${ }^{b, c, d, *}$

${ }^{a}$ Center on Nanoenergy Research, School of Chemistry and Chemical Engineering, School of Physical Science and Technology, Guangxi University, Nanning 530004, P. R. China.

${ }^{b}$ CAS Center for Excellence in Nanoscience, Beijing Key Laboratory of Micro-nano Energy and Sensor, Beijing Institute of Nanoenergy and Nanosystems, Chinese Academy of Sciences, Beijing 101400, P. R. China.

'School of Nanoscience and Technology, University of Chinese Academy of Sciences, Beijing 100049, P. R. China.

${ }^{\mathrm{d} S c h o o l}$ of Materials Science and Engineering, Georgia Institute of Technology, Atlanta, Georgia 30332-0245, USA.

*Correspondence to: zlwang@gatech.edu (Z.L.Wang); puxiong@binn.cas.cn (X.Pu) 


\section{Experimental Section}

\section{Materials}

All reagents were used as received without further purification: PEDOT-PSS $(1.5 \% \mathrm{wt}$. in water, J\&K Scientific), $\mathrm{SnCl}_{2}\left(99 \%\right.$, aladdin), $\mathrm{PdCl}_{2}\left(\mathrm{Pd} 59-60 \%\right.$, aladdin), $\mathrm{NiSO}_{4} \cdot 6 \mathrm{H}_{2} \mathrm{O}(\mathrm{AR}$, Macklin), $\mathrm{NaH}_{2} \mathrm{PO}_{4} \cdot \mathrm{H}_{2} \mathrm{O}$ (AR, Macklin), citric acid (AR, Macklin), and boric acid (AR, Macklin).

\section{Fabrication of Ni-coated textile}

The Ni coated textile was prepared by an electroless plating of Ni. The textile was ultrasound in alcohol for $10 \mathrm{~min}$, then washed with water for 5 minutes and blow-dried. Subsequently, the textile was immersed into $\mathrm{SnCl}_{2}$ solution containing $10 \mathrm{~g} \mathrm{~L}^{-1} \mathrm{SnCl}_{2}$ and $40 \mathrm{~mL} \mathrm{~L}^{-1} 38 \% \mathrm{HCl}$ and $\mathrm{PdCl}_{2}$ solution containing $0.5 \mathrm{~g} \mathrm{~L}^{-1} \mathrm{PdCl}_{2}$ and $20 \mathrm{~mL} \mathrm{~L}^{-1} 38 \% \mathrm{HCl}$ at room temperature for 5 minutes, respectively. Finally, the textile was put into a plating solution for Ni electroless plating at room temperature for 24 hours. The plating solution contains $3.5 \mathrm{~g} \mathrm{NiSO}_{4} \cdot 6 \mathrm{H}_{2} \mathrm{O}, 5 \mathrm{~g}$ $\mathrm{NaH}_{2} \mathrm{PO}_{4} \cdot \mathrm{H}_{2} \mathrm{O}, 3 \mathrm{~g}$ citric acid and $6 \mathrm{~g}$ boric acid dissolved in $200 \mathrm{ml}$ DI water, whose $\mathrm{PH}$ value was adjusted to 8 by using $10 \% \mathrm{NaOH}$ solution.

\section{Fabrication of the PEDOT/Ni-coated textile}

A specific volume of PEDOT-PSS solution was diluted in DI water (ratio of 2:1) with 
ultrasonic treatment for $1 \mathrm{~h}$. Ni-coated textiles were oxygen plasma treated for 30 seconds, and then dipped in as-prepared aqueous dispersion of PEDOT-PSS for 3 minutes. After that, it was dried in an oven at $60{ }^{\circ} \mathrm{C}$ for about 20 minutes. The dip-coating process was repeated for 3 times.

\section{Physical Characterization Methods}

Scanning electron microscope image of the PEDOT/Ni-coated textile was characterized with a Hitachi SU8020. The FTIR spectrum was measured by Fourier transform infrared spectrometer, VERTEX80v. The current-voltage (I-V) curves of the metal semiconductor interface was measured by Keithley 2450 Source Meter. The real-time current and voltage output were recorded in real time by a Keithley 6517B Electrometer, which was controlled by a Labviewbased data acquisition system with a sampling rate of $3000 \mathrm{~s}^{-1}$. Films of PEDOT-PSS prepared by drop-casting method with the PEDOT-PSS aqueous dispersion have been used for fourpoint probe conductivity measurements. 


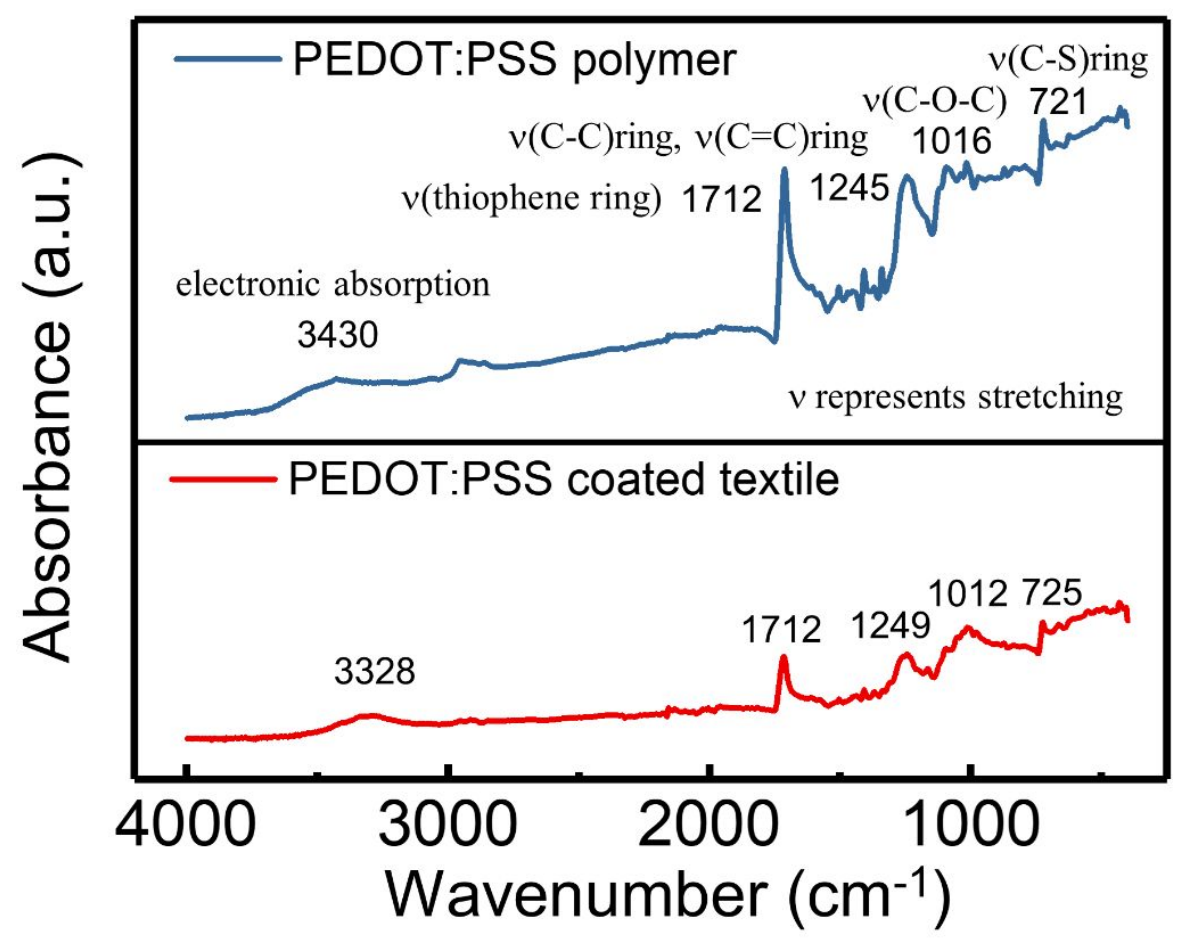

Figure S1. FTIR analysis of the PEDOT-PSS film and the PEDOT/Ni-coated textile.

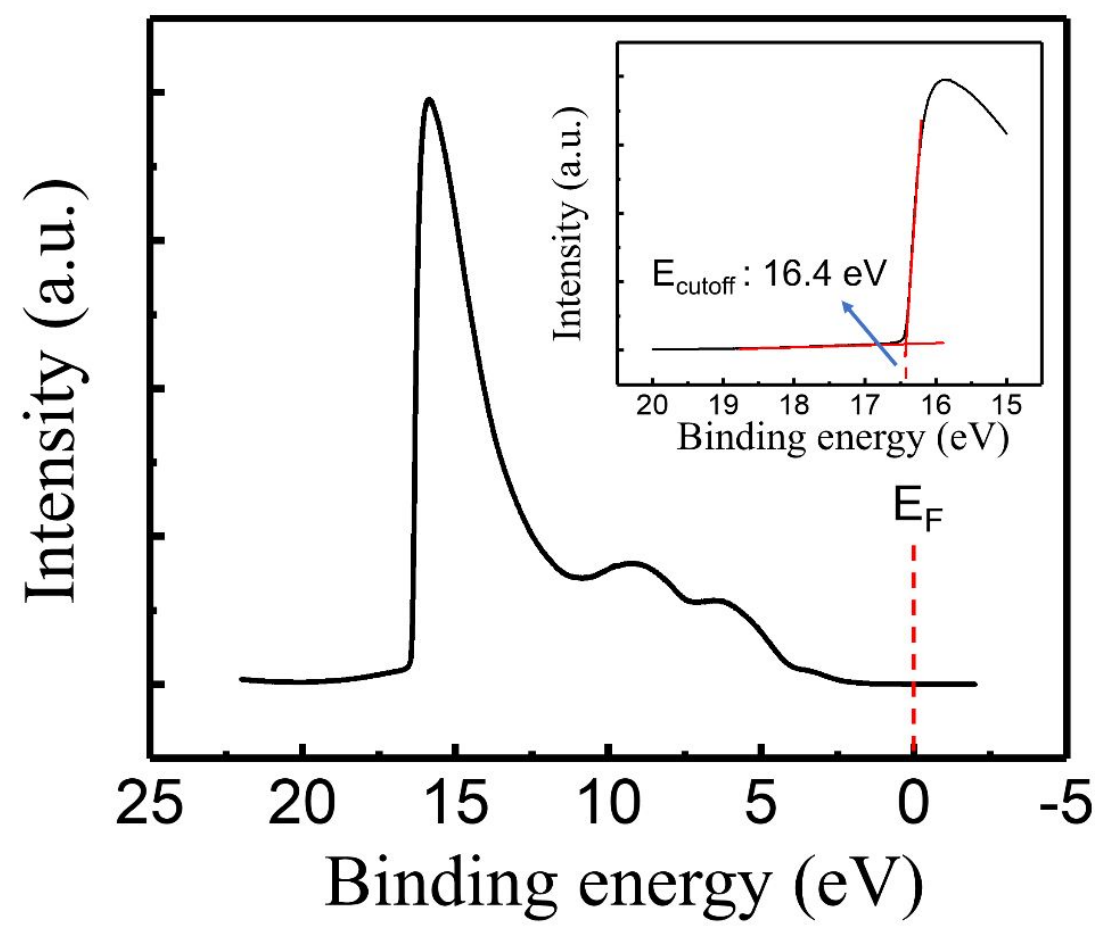

Figure S2. Ups spectra of pristine PEDOT-PSS and secondary electron cutoff region (inset photo). Ultraviolet photoelectron spectroscopy (UPS) with an excitation energy of He I (21.2 
eV) was employed to characterize the electronic structure of the PEDOT-PSS. The work function (WF) of the material surface can be determined using WF $=h v-E_{\text {cutoff. }}{ }^{1}$ Thus, the work functions measured from these spectra for the PEDOT-PSS is $4.8 \mathrm{eV}$.

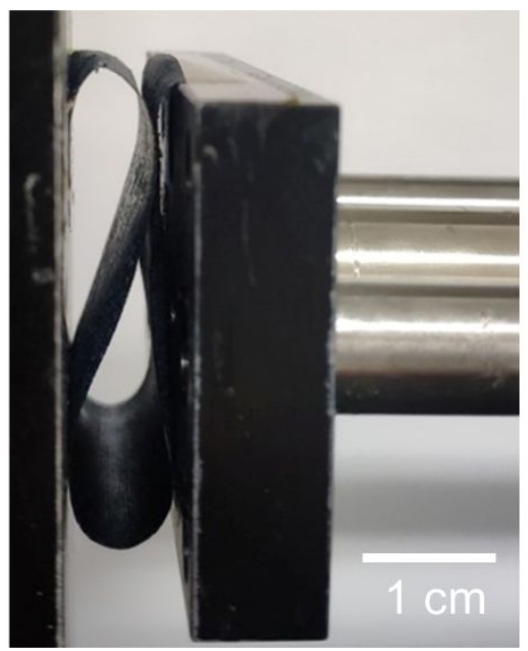

Figure S3. The photograph of the flexible textile at bending state.

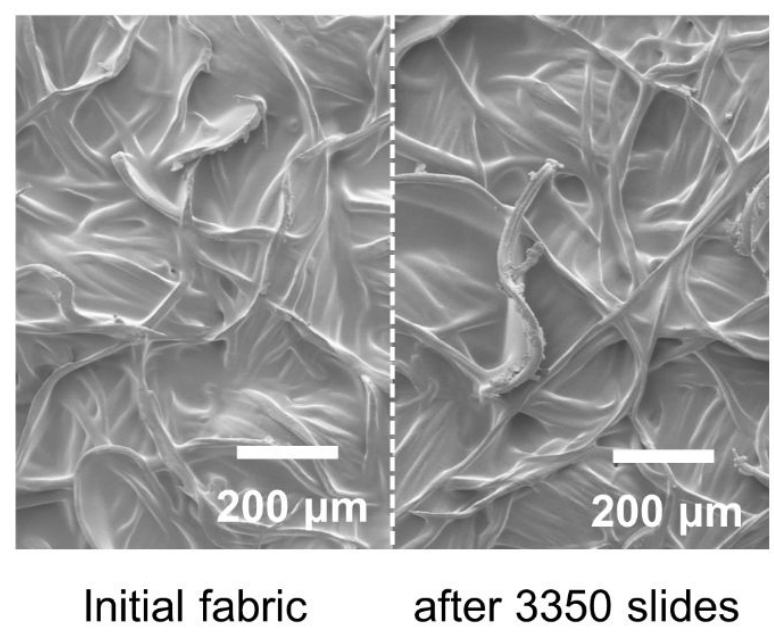

Figure S4. The SEM image of the flexible textile at original state and after 3350 slides. 

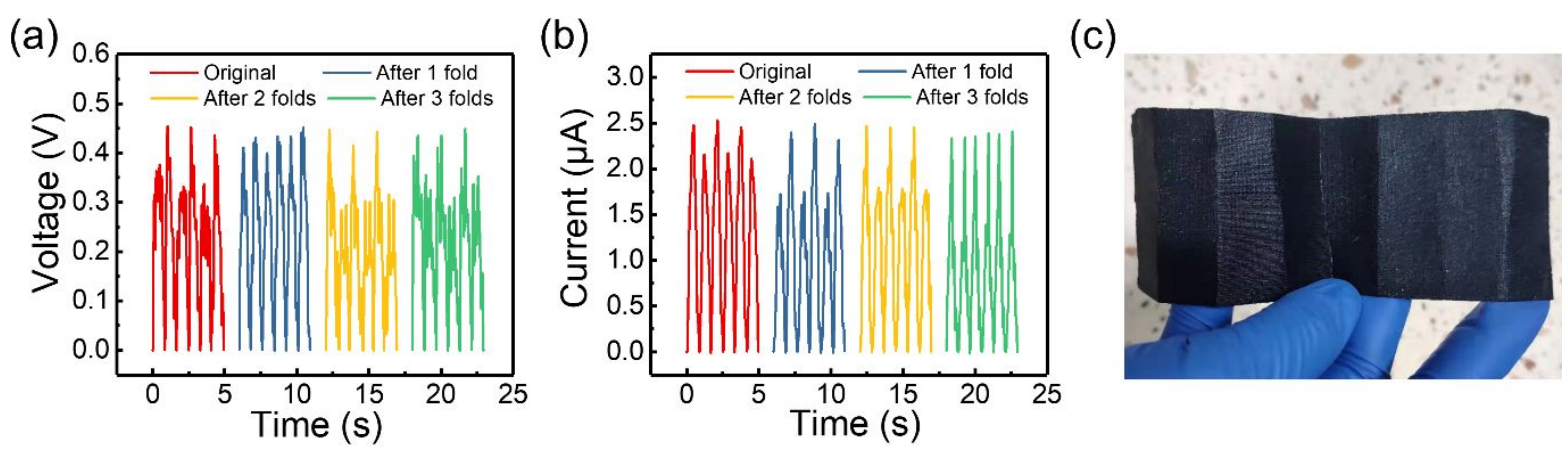

Figure S5. The voltage (a) and current output (b) of the original textile generator and the textile generator after folding one to three times. (c) The picture of the textile after complete folding.

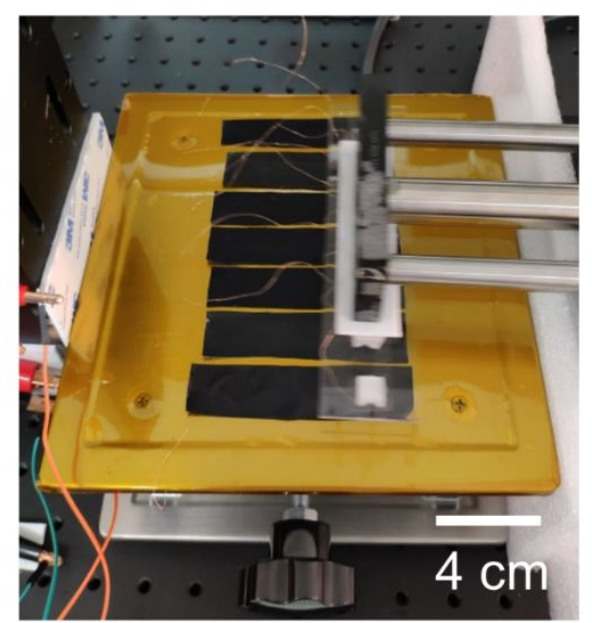

Figure S6. Photograph of seven textile tribovoltaic DC generators connected in series. 
Table S1 A summary of principle, materials and output performances of various DC-TENGs.

\begin{tabular}{|c|c|c|c|c|c|c|c|}
\hline \multirow{3}{*}{ Principles of power generation } & \multirow{3}{*}{ Materials } & Voltage & Current & Current density & Power & \multirow{3}{*}{ Flexibility } & \multirow{3}{*}{ Ref. } \\
\hline & & \multirow[b]{2}{*}{$(\mathrm{V})$} & \multirow[b]{2}{*}{$(\mu \mathrm{A})$} & \multirow[b]{2}{*}{$\left(\mathrm{Am}^{-2}\right)$} & \multirow[b]{2}{*}{$\left(\mathrm{mWm} \mathrm{m}^{-2}\right)$} & & \\
\hline & & & & & & & \\
\hline Contact electrification and dielectric breakdown & PTFE and $\mathrm{Cu}$ & 750 & 15 & $10^{-3}$ & 10 & $\times$ & 2 \\
\hline Phase coupling & PTFE, MC nylon, and $\mathrm{Cu}$ & 380 & 28 & $10^{-3}$ & 38 & $\times$ & 3 \\
\hline Quantum mechanical tunneling & $\mathrm{Pt} / / \mathrm{r}-$ coated AFM probe, $\mathrm{SiO}_{\mathrm{x}}, \mathrm{p}-\mathrm{Si}$ & $0.3-0.4$ & $3-5$ & $1-10$ & $10^{-7}$ & $\times$ & 4 \\
\hline Tribo-tunneling & carbon aerogel and $\mathrm{SiO}_{2} / \mathrm{Si}$ & 2 & 15 & 0.2 & 0.001 & $\times$ & 5 \\
\hline A polymer-inorganic oxide junction & $\mathrm{PPy}, \mathrm{SnO}_{2}, \mathrm{Al}, \mathrm{Au}$ & 0.25 & 0.35 & 0.027 & 0.18 & $\times$ & 6 \\
\hline Tribovoltaic effect on Metal-Semiconductor Interface & Stainless steel and $\mathrm{n}-\mathrm{Si}$ & 0.02 & 20 & 0.1 & 0.0001 & $\times$ & 7 \\
\hline A moving Schottky junction & $\mathrm{Si}, \mathrm{Au}, \mathrm{Al}, \mathrm{Graphene}, \mathrm{Ag}$ & 0.2 & 4 & 40 & 5250 & $\times$ & 8 \\
\hline A dynamic Schottky diode & Fe tip, p-type silicon, Ag & 0.062 & $2.7 \times 10^{4}$ & $2.7 \times 10^{5}$ & $1.2 \times 10^{6}$ & $\times$ & 9 \\
\hline A dynamic $\mathrm{PN}$ junction & P-Si/N-GaAs & 5.1 & 1.5 & 112 & $1.3 \times 10^{5}$ & $\times$ & 10 \\
\hline A sliding Schottky nanocontact & Pt-coated AFM tip, Ag, p-Si, $\mathrm{SiO}_{2}$ & 0.007 & 0.0001 & $10^{6}$ & l & $\times$ & 11 \\
\hline
\end{tabular}


Tribovoltaic effect

Al, PEDOT-PSS, Ni

$0.45-0.7$

2-15

$0.02-0.15$

Abbreviation:

PTFE, polytetrafluoroethylene

MC nylon, mono cast (MC) nylon

PPy, polypyrrole

DI water, deionized water

CAFM, conductive atomic force microscope 


\section{References}

(1) Janardhanam, V.; Yun, H.-J.; Jyothi, I.; Lee, J.; Hong, H.; Rajagopal Reddy, V.; Choi, C.-J., Energy-level alignment and electrical properties of Al/p-type Si Schottky diodes with sorbitol-doped PEDOT:PSS as an organic interlayer. J. Alloys Compd. 2015, 637, 84-89.

(2) Liu, D.; Yin, X.; Guo, H.; Zhou, L.; Li, X.; Zhang, C.; Wang, J.; Wang, Z. L., A constant current triboelectric nanogenerator arising from electrostatic breakdown. Sci. Adv. 2019, 5 (4), eaav6437.

(3) Ryu, H.; Lee, J. H.; Khan, U.; Kwak, S. S.; Hinchet, R.; Kim, S.-W., Sustainable direct current powering a triboelectric nanogenerator via a novel asymmetrical design. Energy Environ. Sci. 2018, 11 (8), 2057-2063.

(4) Liu, J.; Miao, M.; Jiang, K.; Khan, F.; Goswami, A.; McGee, R.; Li, Z.; Nguyen, L.; Hu, Z.; Lee, J.; Cadien, K.; Thundat, T., Sustained electron tunneling at unbiased metal-insulator-semiconductor triboelectric contacts. Nano Energy 2018, $48,320-326$.

(5) Liu, J.; Cheikh, M. I.; Bao, R.; Peng, H.; Liu, F.; Li, Z.; Jiang, K.; Chen, J.; Thundat, T., Tribo-Tunneling DC Generator with Carbon Aerogel/Silicon Multi-Nanocontacts. Adv. Electron. Mater. 2019, 5 (12), 1900464.

(6) Shao, H.; Fang, J.; Wang, H.; Zhou, H.; Lin, T., Direct current energy generators from a conducting polymer-inorganic oxide junction. J. Mater. Chem. A 2017, 5 (18), 8267-8273.

(7) Zhang, Z.; Jiang, D.; Zhao, J.; Liu, G.; Bu, T.; Zhang, C.; Wang, Z. L., Tribovoltaic Effect on Metal-Semiconductor Interface for Direct-Current Low-Impedance Triboelectric Nanogenerators. Adv. Energy Mater. 2020, 10 (9), 1903713.

(8) Lin, S.; Lu, Y.; Feng, S.; Hao, Z.; Yan, Y., A High Current Density DirectCurrent Generator Based on a Moving van der Waals Schottky Diode. Adv. Sci. 2019, 31 (7), e1804398.

(9) Lin, S.; Shen, R.; Yao, T.; Lu, Y.; Feng, S.; Hao, Z.; Zheng, H.; Yan, Y.; Li, E., Surface States Enhanced Dynamic Schottky Diode Generator with Extremely High Power Density Over 1000 W m(-2). Adv. Sci. 2019, 6 (24), 1901925.

(10)Lu, Y.; Hao, Z.; Feng, S.; Shen, R.; Yan, Y.; Lin, S., Direct-Current Generator Based on Dynamic PN Junctions with the Designed Voltage Output. iScience 
2019, 22, 58-69.

(11)Liu, J.; Goswami, A.; Jiang, K.; Khan, F.; Kim, S.; McGee, R.; Li, Z.; Hu, Z.; Lee, J.; Thundat, T., Direct-current triboelectricity generation by a sliding Schottky nanocontact on MoS2 multilayers. Nat. Nanotechnol. 2018, 13 (2), 112-116.

(12)Lin, S.; Chen, X.; Wang, Z. L., The tribovoltaic effect and electron transfer at a liquid-semiconductor interface. Nano Energy 2020, 76, 105070.

(13)Zheng, M.; Lin, S.; Xu, L.; Zhu, L.; Wang, Z. L., Scanning Probing of the Tribovoltaic Effect at the Sliding Interface of Two Semiconductors. Adv. Mater. 2020, 32 (21), e2000928. 